\title{
Let there be light
}

\author{
Inorganic semiconductors, organic emitters and colloidal quantum dots are pushing the LED revolution \\ in lighting and displays.
}

From solar panels and telescopes to laser-surgery tools and fibre cables, many life-changing innovations have arisen from the fundamental discoveries enabled by the exploration of light's interaction with matter. The United Nations has acknowledged the role of light by proclaiming 2015 the International Year of Light and Light-based Technologies ${ }^{1}$, an event also celebrated by the Nature journals through selections of research articles and opinion pieces illustrating scientific contributions to the understanding and mastering of light ${ }^{2}$. Out of these, efficient, blue light-emitting diodes (LEDs), developed by the 2014 Nobel laureates Isamu Akasaki, Hiroshi Amano and Shuji Nakamura ${ }^{3}$, are one of the most important. In this issue, we explore the potential of a broad range of materials for LED applications, and discuss the impact of this technology in our society.

The possibility to generate light from an electric arc or from an incandescent filament revolutionized the lighting industry in the nineteenth century, and incandescent bulbs and fluorescent tubes are today leading technologies in lighting applications. However, the performance of these devices, in terms of efficiency (the amount of electrical energy converted into useful light) and quality of emitted light, respectively, is far from optimal. This is where LEDs come into play. These devices rely on electroluminescence, an extremely efficient light-generation process first observed in 1907 and based on the recombination of electric charges injected in a semiconductor. Yet, as Philipp Pust, Peter Schmidt and Wolfgang Schnick discuss in a Commentary on page 454 , it took several decades to understand how to make high-performance light sources that use this process. The crystalline quality of the semiconductors composing the device and the possibility to modify their charge transport properties by introducing dopant atoms turned out to be crucial for the performance of inorganic LEDs; but both factors were difficult to control in large-bandgap materials emitting in the blue spectral range. A breakthrough came from the research of the three Nobel laureates, who developed a growth process based on metal-organic chemical vapour deposition and a post-growth thermal treatment, which allowed the fabrication,

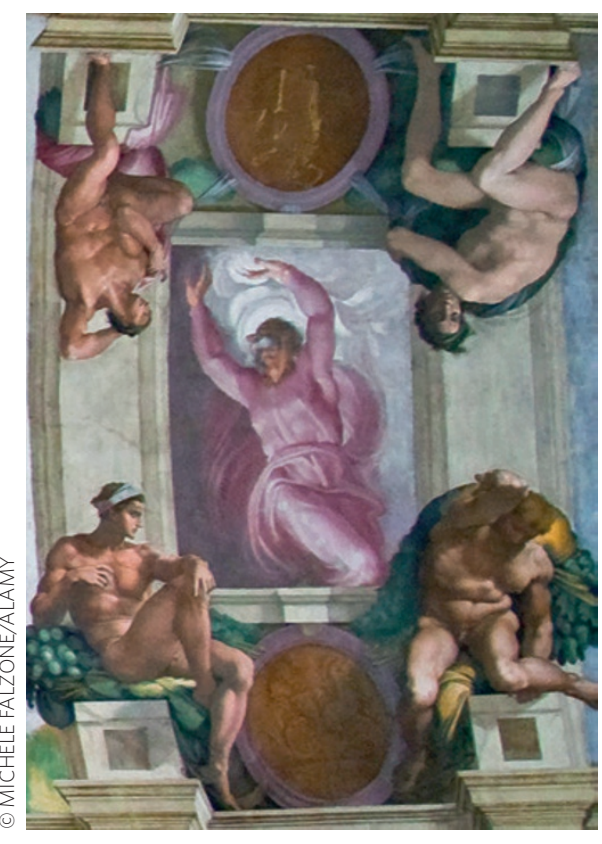

Figure 1 | Separation of Light from Darkness, one of Michelangelo's frescoes on the ceiling of the Sistine chapel, now illuminated with LEDs.

in 1994, of bright blue LEDs based on GaN compounds.

The high-energy blue and ultraviolet light obtained with these compounds is beneficial for several applications, such as high-density optical storage (the blu-ray disc technology) ${ }^{4}$ and sterilization processes. Importantly, blue LEDs enable the realization of extremely efficient and long-lasting white light sources, either by including phosphors that convert part of the blue radiation to green, yellow and red light, or by combining the devices with GaP-based LEDs. A proper selection of the materials allows the emission properties to be optimized for specific lighting applications. For example, the Sistine chapel in Rome ${ }^{5}$ has been illuminated with customized white LEDs to minimize light-induced damage to the 500-year-old frescoes decorating the walls and ceiling (Fig. 1). The so-called solid-state lighting revolution is expected to significantly reduce the energy requirements for lighting applications (which represent at least $20 \%$ of the electrical energy consumption in industrial economies), with consequent beneficial reductions in greenhouse gas emissions ${ }^{3}$.

As soon as the first sparks of electroluminescence from organic semiconductors ${ }^{6}$ and colloidal quantum $\operatorname{dots}^{7}$ were observed, organic LEDs (OLEDs) and quantum dot LEDs (QD-LEDs) also entered the race for solid-state lighting. At present, the overall efficiency and average lifetime of these technologies lag behind those of inorganic LEDs. Yet, as noted by Sebastian Reineke in a Commentary on page 459, OLEDs and QD-LEDs can leverage other strengths, such as their compatibility with large-area deposition and patterning techniques and with a broad range of substrates, including flexible supports. Also, ultrathin, large OLED panels can provide the same light output as a compact LED while reducing the brightness per unit area, resulting in a glarefree light source that is pleasant to the eye and compatible with artistic applications. Beyond lighting, flat OLEDs are already an established reality in the displays market, where OLEDs and liquid-crystal displays (LCDs) backlit by white inorganic LEDs compete in terms of offering superior picture quality and long-term stability for a broad range of display sizes. Although it may take some time for commercial examples of QD-LED displays to reach the market, the excellent performance of quantum dots as fluorescent emitters combined with the high efficiency of blue GaN LEDs has recently been harnessed to make displays with enhanced colour purity ${ }^{8}$

Without doubt, the current performance of solid-state sources is a significant leap forward from incandescent bulbs, and further technological and materials optimization is likely to lead to less expensive and more efficient devices with extended lifetimes. LEDs offer bright hopes for a more sustainable future.

\footnotetext{
References

1. http://www.light2015.org/Home.htm

2. http://www.nature.com/collections/kwhbfh

3. http://www.nobelprize.org/nobel_prizes/physics/laureates/2014/ advanced-physicsprize2014.pdf

4. Nature Mater. 9, 372-373 (2010).

5. http://www.led4art.eu/

6. Tang, C. W. \& VanSlyke, S. A. Appl. Phys. Lett. 51, 913-915 (1987)

7. Colvin, V. L., Schlamp, M. C. \& Alivisatos, A. P. Nature 370, 354-357 (1994).

8. Nature Nanotech. 9, 325 (2014)
} 